\title{
Anti-infective activity of immunomodulators
}

\author{
K. Noel Masihi
}

\section{Introduction}

Infectious diseases continue to cause suffering and death in this teeming world of almost 6 billion people. Every individual is vulnerable to microbial infections regardless of socioeconomic status, gender, age group or ethnic background. Increased international air travel, mass tourism and the expansion of globalization have meant that disease-producing pathogens can be transported and spread rapidly from one geographic region to another. This has been dramatically demonstrated by the sudden and completely unexpected multi-country outbreak of severe acute respiratory syndrome (SARS) in the beginning of 2003 [1]. This atypical pneumonia, caused by a human coronavirus, led the World Health Organization to issue an exceptional global alert and caution against travel to certain countries. The unfolding tragedy of AIDS, particularly in many developing nations, continues to be vividly highlighted by the scientific and public media. According to current estimates, over 30 million people are virus carriers and around 15000 new cases of HIV occur daily. Tuberculosis and other infections which caused ravages in the nineteenth century are once again resurgent. Several infectious agents, such as neurological variants of Creutzfeldt-Jakob disease in Europe, vector-borne West Nile virus in the USA, vancomycin-resistant forms of bacteria in Japan, and virulent avian influenza in humans in Hong Kong, have emerged as public health concerns over the past few years. The sheer adaptability and the destructiveness of infectious microbes pose threats of unforeseen diseases in the 21st century. According to the World Health Organization, at least 30 new diseases have emerged over the past two decades and underscore the need to be vigilant.
Antimicrobial drugs have been instrumental in saving the lives of millions of people worldwide. The antibiotic magic cure of the previous 50 years is, however, being steadily eroded by the emergence of DRUG-RESISTANT microorganisms [2]. This is shown by the adverse effects on the control and treatment of deadly diseases caused by Mycobacterium tuberculosis and Plasmodium falciparum. The acute respiratory infections in children, mostly caused by Pneumococci and Haemophilus influenzae, are becoming more drug-resistant. Over 90\% of Staphylococcus aureus strains and about $40 \%$ of Pneumococci strains are resistant to penicillin which was introduced in the 1940s. Salmonella typhi, Streptococcus pneumoniae, Enterococcus faecium, and Shigella dysenteriae have been reported as multidrug-resistant. Crucial drug choices for the treatment of common infections by bacteria, viruses, parasites, and fungi are becoming limited and even nonexistent in some cases. This development has not been parallelled by an effective increase in the discovery of new medicines for most microbial pathogens [3]. The struggle to control infectious diseases, far from being over, has acquired a new poignancy. Novel concepts acting as adjuncts to established therapies are urgently needed.

The immune system can be manipulated specifically by vaccination or nonspecifically by immunomodulation. IMMUNOMODULATORS include both immunostimulatory and immunosuppressive agents. This chapter will concentrate on immunostimulatory agents capable of enhancing host defence mechanisms to provide protection against infections. Synonymous terms for IMMUNOMODULATORS include biological response modifiers, immunoaugmentors, immunostimulants or immunorestoratives. Their mode of action includes augmentation of the antiinfectious immunity by the cells of the immune sys- 


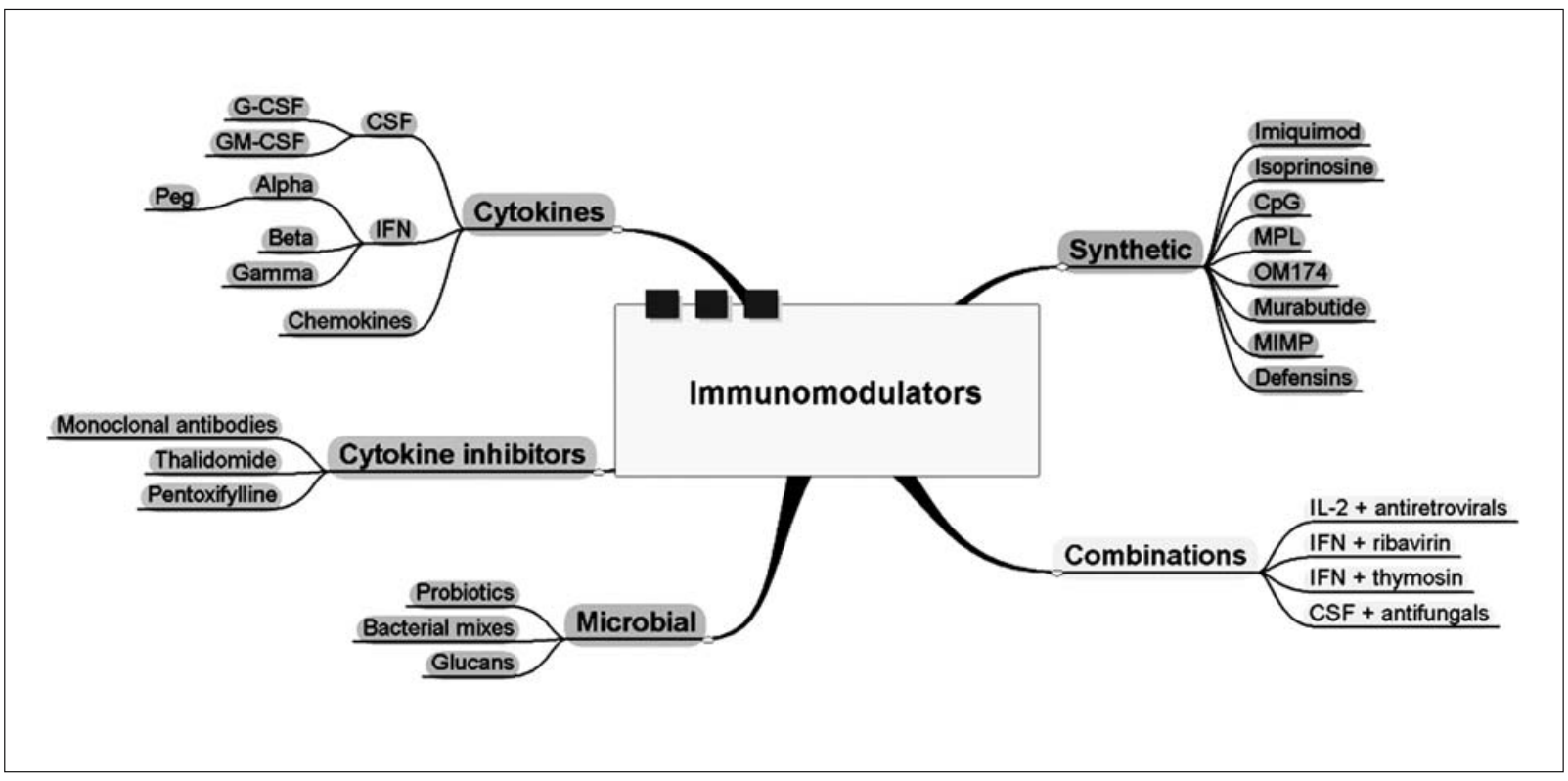

FIGURE 1. IMMUNOMODULATORS

tem encompassing lymphocyte subsets, MACROPHAGES, dendritic and natural killer (NK) cells. Further mechanisms can involve induction or restoration of immune effector functions and tilting the balance towards CYTOKINE pathways germane to protection. A diverse array of recombinant, synthetic, and natural IMMUNOMODULATORY preparations for prophylaxis and treatment of various infections are available today [4-6]. A concise mind-map of the IMMUNOMODULATORS discussed in this chapter is summarised in Figure 1.

\section{Cytokine immunomodulators}

The interactions between a host and an infectious pathogen are complex, diverse and intricately regulated. CYTOKINES, hormone-like polypeptides possessing pleiotropic properties, are crucial in orchestrating the appropriate immune responses critical for the outcome of an infection. Certain CYTOKINES stimulate the production of other CYTOKINES and interact in synergistic or antagonistic networks. CYTOKINES exhibit specific IMMUNOMODULATORY properties that can enable manipulation of the host response to enhance overall immunogenicity and direct the nature of the response either toward a type 1 or type 2 pathway. In the type 1 response, Th1 cells produce interferon- $\gamma$ (IFN- $\gamma$ ), tumor necrosis factor- $\beta$ (TNF- $\beta$ ) and INTERLEUKIN- (IL-) 2 that are required for effective development of cell-mediated immune responses to intracellular microbes. In the type 2 response, Th2 cells produce IL-4 and IL-5 that enhance humoral immunity to T-dependent ANTIGENS and are necessary for immunity to helminth infections. Recent studies have shown that lymphoid progenitor-derived DENDRITIC CELLS (DC1) can also express IL-12 and preferentially induce type $1 \mathrm{~T}$ CELL responses, whereas myeloid progenitor-derived DENDRITIC CELLS (DC2) express IL-10 and induce type 2 T CELL responses. Local and systemic effects of CYTOKINES are, thus, intimately involved in the host control of infections (Fig. 2) (also see chapters A4, A7 and C5). Several recombinant and natural CYTOKINE preparations such as INTERFERONS, granulocyte COLONY-STIMULATING FACTOR, and IL-2 are already licensed for use in patients. 


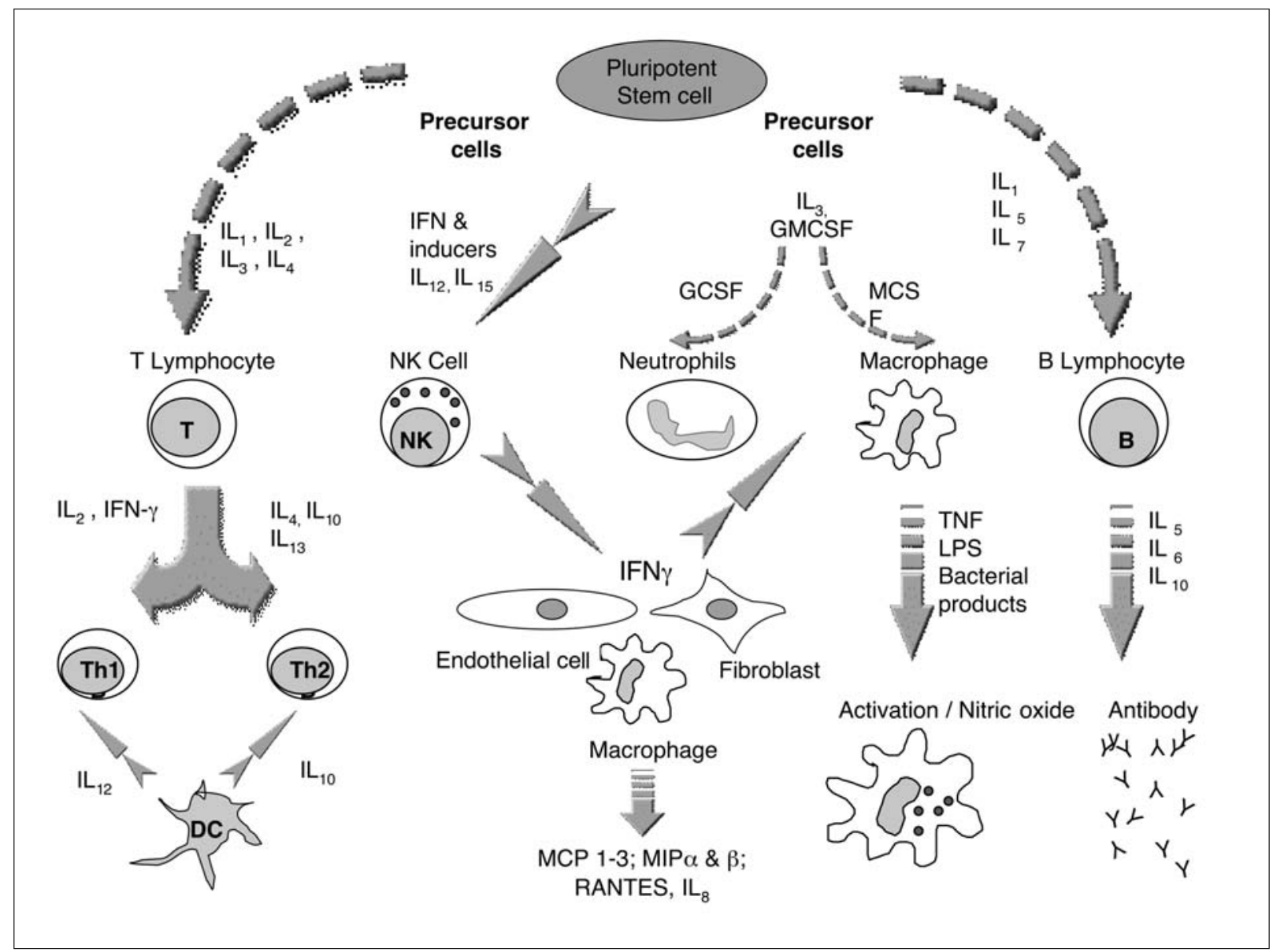

FIGURE 2. INTERACTION OF IMMUNE SYSTEM CELLS AND CYTOKINES

\section{Interferons and combinations}

INTERFERONS play an important role in host defense against infectious pathogens and in the regulation of immune responses. INTERFERON- $\alpha$ (IFN- $\alpha$ ) is a clinically effective therapy used in a wide range of viral infections besides its application in malignant melanoma, basal cell carcinoma and warts. Natural IFN- $\alpha$ obtained from human serum and LEUKOCYTES is currently licensed for the treatment of a rare form of cancer, hairy cell leukemia. Recombinant IFN- $\alpha 2 \mathrm{a}$ is licensed for treatment of chronic active hepatitis $\mathrm{B}$ and for HePATITIS C VIRUS infections. However, only a small subset of patients with hepatitis B and around $40 \%$ of cases with hepatitis C are generally responsive to interferon therapy. IFN- $\alpha$ is also approved for treating condyloma acuminata caused by human papilloma virus and for Kaposi sarcoma in patients with HIV infection.

HePATITIS B VIRUS (HBV) infects around 400 million people and kills between 1 and 2.5 million people a year. Overall, 15 to $25 \%$ of HBV carriers die from chronic hepatitis, cirrhosis or hepatocellular carcinoma. At present, only two licensed therapies for treating liver disease caused by infection with the HBV are available. These are IFN- $\alpha$ and lamivudine. 
Several promising studies have shown the effectiveness of lamivudine-interferon (LAM/IFN) COMBINATION THERAPY and it is gaining increasing favour in the treatment of chronic hepatitis B $[7,8]$.

Thymosin- $\alpha 1$ (T- $\alpha 1)$ influences T-cell maturation, production of Th1-type CYTOKINES, and activity of NK cell-mediated cytotoxicity [9]. Patients with chronic hepatitis B treated with T- $\alpha 1$ had augmented NKT CELLS and $\mathrm{CD} 8^{+}$cytotoxic T LYMPHOCYTES in the liver. Forty-eight weeks after T- $\alpha 1$ treatment some patients showed normalized alanine aminotransferase (ALT) and decreased HBV-DNA to undetectable level from serum [10]. The combination of $\mathrm{T}-\alpha 1$, ribavirin and either PEGYLATED IFN- $\alpha,-\alpha 2 \mathrm{a},-\beta$ and $-\gamma$ are stated to simultaneously and substantially reduce or eliminate the side-effects normally associated with the administration of IFN alone.

Hepatitis C Virus (HCV) infection is a common cause of chronic viral liver disease and is a leading indication for liver transplantation. Almost all such patients show recurrent hepatitis C viremia. Progressive fibrosis and cirrhosis after liver transplantation have been observed with some of the patients progressing to cirrhosis within 5 years of transplantation. Genotype 1 is considered to be the most resistant to therapy, whereas genotypes 2 and 3 are more responsive to therapy; genotype 4 seems to be similar to genotype 1 in this respect (Box 1). IFN- $\alpha 2 b$ plus ribavirin COMBINATION THERAPY has been found to be effective in chronic HCV patients with genotypes 2 and 3 who are virological nonresponders to IFN monotherapy [11]. The treatment duration is 48 weeks for genotype 1 and 24 weeks for other genotypes.

Standard IFN- $\alpha$ has the drawbacks of a short serum half-life and rapid clearance. To overcome this problem, PEGYLATED forms of IFN have been developed and tested clinically. Pegrlated IFN- $\alpha 2 b$ is formed by covalent conjugation of a $12-\mathrm{kd}$ monomethoxy polyethylene glycol (PEG) molecule to IFN- $\alpha 2 b$, and PEGYLATED IFN- $\alpha 2 \mathrm{a}$ by covalent conjugation of a 40-kd branched monomethoxy PEG molecule to IFN- $\alpha 2 \mathrm{a}$. PEGYLATED IFN- $\alpha 2 \mathrm{~b}$ has a prolonged serum half-life (40 hours) relative to standard IFN$\alpha 2 b$ (7-9 hours). The greater polymer size of PEGYLATED IFN- $\alpha 2 \mathrm{a}$ acts to reduce glomerular filtration, markedly prolonging its serum half-life (72-96 hours) compared with standard IFN- $\alpha 2$ a (6-9 hours). Peginterferon $\alpha 2 \mathrm{a}$ (40 KD) has superior virological EFFICACY to IFN- $\alpha 2 \mathrm{a}$, and elicits histological improvements in chronic HCV genotype 1 patients with and without sustained virological response. Peginterferon $\alpha 2 \mathrm{a}(40 \mathrm{KD})$ is effective in patients infected with viral genotype 1 and those with liver cirrhosis. The addition of ribavirin to peginterferon $\alpha$ 2a (40 KD) further enhances the therapeutic benefit for patients with HCV [12]. Once-weekly dosing with either PEGYLATED IFN- $\alpha 2 a$ Or PEGYLATED IFN- $\alpha 2 b$ has been shown to produce significantly higher rates of viral eradication than standard thrice-weekly IFN- $\alpha$ therapy.With respect to the treatment of chronic hepatitis $\mathrm{C}(\mathrm{CHC})$, the greatest anti-HCV efficacy has been achieved with the combination of once-weekly PEGYLATED IFN and ribavirin [13]. The use of ribavirin and PEGYLATED IFN- $\alpha$, particularly PEGYLATED IFN- $\alpha 2 b$, for the treatment of $\mathrm{CHC}$ infections, which involves a first and a second treatment time period, each of at least 20 to 30 weeks, has been reported. In clinical studies to assess sustained loss of serum HCV-RNA 12 weeks post-treatment, a regimen employing ribavirin plus PEGYLATED IFN- $\alpha 2 \mathrm{~b}$ for 48 weeks gave a successful response in $54 \%$ of patients, with a $41 \%$ response in those with HCV genotype 1.

INTERFERON- $\beta$ (IFN- $\beta$ ) obtained from human FS-4 fibroblast cell lines is licensed for use in severe uncontrolled virus-mediated diseases in cases of viral encephalitis, herpes zoster and varicella in immunosuppressed patients. A further indication is viral infection of the inner ear with loss of hearing. The standard treatment for multiple sclerosis, a disease without a definitively elucidated etiology, is currently with IFN- $\beta$.

INTERFERON- $\gamma$ (IFN- $\gamma)$ is the major mediator of host resistance during the acute and chronic phases of infection and is pivotal in protection against a variety of intracellular pathogens. IFN- $\gamma$ is produced by both $\mathrm{CD}^{+}$and $\mathrm{CD} 8^{+} \mathrm{T}$ CeLLS and can induce class I and class II MHC products. Patients with chronic granulomatous disease are unable to generate an oxidative respiratory burst. As a consequence, they develop recurring catalase-positive bacterial infections such as S. aureus, Pseudomonas cepacia, and Chromobacterium violaceum. Multicenter clinical trials have shown that sustained administration of IFN- $\gamma$ to 


\section{Box 1. Hepatitis C VIRUS GenOtypes \\ Genotype \\ Sensitivity to interferon therapy \\ 1 resistant \\ 2 responsive \\ 3 responsive \\ $4 \quad$ resistant}

chronic granulomatous disease patients markedly reduced the relative risk of serious infection. IFN- $\gamma$ is licensed as a therapeutic adjunct for use in patients with chronic (septic) granulomatosis for reduction of the frequency of serious infections. Actimmune ${ }^{\mathrm{TM}}$, a IFN- $\gamma$ preparation, is marketed for chronic granulomatous disease, mycobacterial and fungal infections.

The major side-effects of all interferon therapies include flu-like syndromes, fever, myalgia, headache and fatigue. Hypotension, granulocytopenia, and thrombocytopenia can also occur. Deleterious effects on the central nervous system (CNS), particularly at high doses, have been observed.

\section{Colony-stimulating factors}

A high incidence of HIV-infected patients have neutropenia, which considerably increases risk for bacterial and fungal infections. G-CSF preparations such as Filgrastim (r-metHuG-CSF) can significantly enhance NEUTROPHIL functions in patients with AIDS [14] and reverse neutropenia associated with HIV and cytomegalovirus (CMV) infections. Filgrastim has been granted a license extension to cover the treatment of persistent neutropenia at an advanced stage of HIV infection. In one study, Filgrastim-treated patients have been shown to have 54\% fewer severe bacterial infections and 45\% fewer days in hospital for any bacterial infections [15]. Determination of absolute numbers of CD34+ progenitor cells and progenitor cell function in HIV-infected patients showed that G-CSF mainly increases the number and differ- entiation of myeloid progenitors. G-CSF and GM-CSF are used to reverse leukopenia as adjunctive therapy for HIV-associated infections. In a study conducted at 27 European centers on AIDS patients with CMV infection, G-CSF (lenograstim) was found to be suitable for the treatment of ganciclovir-induced neutropenia [16]. Multiple deficiencies are involved in the progression of fungal infections in neutropenic and nonneutropenic cancer patients. Although clinical experience is still limited, G-CSF, GM-CSF, and macrophage CSF show promise as adjuvant therapy for fungal infections [17].

\section{Emerging cytokine therapies}

\section{IL-2 combinations}

Highly active antiretroviral therapy (HAART), a combination of nucleoside and non-nucleoside reverse transcriptase, protease and fusion drug inhibitors, has improved the morbidity and survival of patients with HIV infection. Currently available antiretroviral agents can suppress viral replication and partially reverse cellular immunity defects in HIV patients. Combination antiretroviral drug therapy has been pivotal in the beneficial outcome in many HIV-1 infections and has rekindled a renewed interest in the use of IMMUNOTHERAPY as an adjunct treatment $[18,19]$. The ability of IL-2 to induce expansion of the $\mathrm{CD} 4^{+} \mathrm{T}$ lymphocyte pool has made it the most studied CYTOKINE in the treatment of HIV infection. Initial studies of high-dose IL-2 therapy were reported over a decade ago but were severely limited by toxicity problems. The current approach of decreasing the IL-2 amounts and using intermittent dosing has dramatically increased $\mathrm{CD}^{+}$cell counts in HIV-infected persons. A majority of recent combinatory studies in HIV have, thus, been been performed with IL-2 and HAART. Immune-based therapy with IL-2 when used as adjunctive therapy to antiretroviral therapy may further improve immune responses, as demonstrated by an increase in $\mathrm{CD} 4^{+} \mathrm{T}$-lymphocyte counts in recent clinical trials [19]. An IL-2 regimen consisting of 5-day cycles administered every 4-8 weeks aims at expanding the $\mathrm{CD} 4^{+} \mathrm{T}$ lymphocyte pool. 
Combined antiretroviral treatment in some HIVinfected persons does not lead to a rapid increase in CD4 cell counts. Patients with CD4 cell counts $<200$ cells after 9 or more months of combined antiretroviral treatment received IL-2 IMMUNOTHERAPY $(4.5 \times$ $10^{6} \mathrm{IU}$ twice daily for 5 days every 6 weeks). After only 3 cycles, their CD 4 cell counts increased significantly (mean cell count from 123 cells to 229 cells $/ \mathrm{mm}^{3}$ ). An increase in the naive CD45RA+ T CELL subset was noted. IL-2 IMMUNOTHERAPY appears to accelerate the recovery of $\mathrm{CD} 4^{+}$LYMPHOCYTES in persons whose $\mathrm{CD}^{4}+$ cell counts fail to increase rapidly in response to combined antiretroviral treatment [20].

\section{Chemokines and chemokine modulators}

CHEMOKINES have been historically regarded as leukocyte chemoattractants capable of regulating cellular trafficking into inflammatory sites. The term chemokine is abbreviated from chemotactic CYTOKINES. The major classes of CHEMOKINES are constituted by the CXC or $\alpha$ CHEMOKINES, the CC or $\beta$ CHEMOKINES, the $\mathrm{C}$ or $\gamma$ CHEMOKINES, and the CX3C CHEMOKINES. Accumulating evidence suggests that CHEMOKINES have a broad range of functions, including macrophage activation, NEUTROPHIL degranulation, dendritic cell maturation, T CELL activation and B CELL ANTIBODY class switching. Thus, CHEMOKINES can influence both the innate and acquired phases of an immune response. CHEMOKINES can be mediators of angiogenesis and also play important roles in the development of the immune, circulatory and central nervous system. Currently, the chemokine family has expanded to nearly 50 ligands; 16 CXCL, 28 CCL, 2 XCl and 1 CX3CL CHEMOKINES have been identified. Furthermore, around 18 chemokine receptors, including 6 CXCR, 10 CCR, 1 XCR and 1 CX3CR, have been described (also see Chapter A.4).

The human immunodeficiency virus (HIV) can infect a wide range of human cells but has a particular tropism for $\mathrm{CD}^{+} \mathrm{T}$ CELLS and the MONOCYTEMACROPHAGE cell lineage [21]. HIV induces T-cell dysfunction and CD8 ${ }^{+} \mathrm{T}$ CELL apoptosis, decreasing the number of T-cells that ultimately leads to immunodeficiency. B-cell dysfunction can be caused by HIV and is characterized by hypergammaglobulinemia, polyclonal activation, and absence of specific ANTIBODY responses. HIV entry into the host cell is mediated through the CD4 receptor and a variety of coreceptors. Chemokine receptors-mainly CCR5 and CXCR4-have been discovered to be necessary for HIV entry [22-25]. Binding of the HIV-1 envelope glycoprotein gp120 to CD4 and an appropriate chemokine receptor triggers conformational changes facilitating the fusion of the viral and host cell membranes. HIV-1 found in the vagina, rectal mucosa, CNS and in resident MACROPHAGES of many other tissues is mainly CCR-5-dependent. Macrophage-tropic (R5) HIV-1 variants predominantly make use of the CCR5 coreceptors [26,27].The T-celltropic (X4) and dualtropic (R5X4) HIV-1 strains, generally associated with the clinical manifestations of AIDS, emerge after a latency of several years, although pathogenesis of the CNS and related symptoms are normally associated to M-tropic (R5) HIV-1 strains. X4 HIV-1 infection augments the expression of CHEMOKINES such as MIP-1 $\alpha$ (macrophage inflammatory protein) and RANTES (regulated upon activation normal T CELL expressed and secreted).

HIV infection can be inhibited by CHEMOKINES and chemokine-related molecules that are ligands for those chemokine receptors that function as coreceptors. Chemokine receptors, thus, represent important targets for intervention in HIV and the search for molecules that have a therapeutic potential as inhibitors of these receptors has been intense [28-30]. Chemokine receptors belong to the rhodopsin family of the G-protein-coupled receptor family. Intervention strategies based on chemokine antagonists that could be useful for the therapeutics of HIV include receptor-ligand interaction, prevention of the chemokine-glycosaminoglycan interaction, interfering with the signalling pathways that are induced upon receptor activation, and modification of receptor pathways [31,32].

Azaheterocyclylcarboxamide, piperidinyl, and pyrrolidine compounds and their derivatives targeting CCR-5 have been advocated recently for the treatment of AIDS. $\beta$-CHEMOKINES such as RANTES exhibit clear-cut suppressive effects on HIV replication in peripheral blood mononuclear cells of HIV-infected individuals. CCR5 can be blocked and/or downmod- 
ulated by RANTES and by antibodies to three extracellular domains of CCR5. Recently, modified CHEMOKINES and small derivative molecules maintaining the HIV-inhibitory function, but devoid of receptor-activating capability, have been generated [33].

\section{Cytokine inhibitors}

Many infectious diseases, including HIV and malaria, and chronic inflammatory conditions can induce deleterious overproduction of proinflammatory CYTOKINES such as TNF- $\alpha$ and IL-1. This has generated intense interest in developing agents that can block the activity of such CYTOKINES. Inhibiting TNF activity has been singularly successful in the treatment of AUTOIMMUNE DISEASES. Monoclonal antibodies including adalimumab (Humira; Abbott), etanercept (Enbrel; Amgen/Wyeth), and infliximab (Remicade; Centocor) are being used in the treatment of rheumatoid arthritis. An INTERLEUKIN-1 (IL-1) receptor antagonist, Kineret, has also been recently licensed. Another potent TNF inhibitor, thalidomide, has been used in trials in HIV patients. Structural analogues of thalidomide with improved TNF- $\alpha$ inhibitory activity are currently being developed. Pentoxifylline, a methylxanthine usually used in the treatment of peripheral arterial circulatory disorders, has been shown to inhibit TNF synthesis. Currently, clinical trials are ongoing with phosphodiesterase inhibitors and small-molecule TNF-converting enzyme that specifically interrupt the signalling pathways of TNF.

\section{Synthetic immunomodulators}

\section{Imiquimod derivatives}

TOLL-LIKE RECEPTORS (TLRs) are type I transmembrane proteins that recognize microbial conserved structures involved in INNATE IMMUNITY (Box 2). Recent studies have shown that TLR3 recognizes dsRNA, a viral product, whereas TLR9 recognizes unmethylated $\mathrm{CpG}$ motifs frequently found in the genome of bacteria and viruses, but not vertebrates. In humans, TLR7 and TLR9 are expressed on the plasmacytoid DENDRITIC CELLS (PDC) which can rapidly synthesize

$\begin{array}{ll}\text { BOX 2 } & \text { TOLL-LIKE RECEPTORS } \\ \text { TLR } & \text { Ligands } \\ \text { TLR1 } & \text { Lipoprotein } \\ \text { TLR2 } & \text { Lipoprotein, peptidoglycan } \\ \text { TLR3 } & \text { dsRNA } \\ \text { TLR4 } & \text { Lipopolysaccharide, lipoteichoic acid } \\ \text { TLR5 } & \text { Flagellin } \\ \text { TLR6 } & \text { Lipoprotein } \\ \text { TLR7 } & \text { Imiquimod } \\ \text { TLR8 } & \text { Imiquimod } \\ \text { TLR9 } & \text { Unmethylated CpG } \\ \text { TLR10 } & ?\end{array}$

large amounts of IFN- $\alpha$ and IFN- $\beta$ in response to viral infection. These observations indicate that TLR3, TLR7 and TLR9 may play an important role in combating viral infections. Interestingly, TLR7 recognizes synthetic IMMUNOMODULATORS such as imidazoquinolone compounds that are used against viral infections.

IMIQUIMOD is a fully synthetic immune response enhancing imidazoquinoline amine, (S-26308, R-837) (1-(2-methylpropyl)-1H-imidazo[4,5-c] quinolin-4 amine) [34]. IMIQUIMOD is marketed as Aldara ${ }^{\mathrm{TM}}$ for genital warts, but is widely used for basal cell carcinoma, actinic keratosis and molluscum contagiosum. It has shown promise in lentigo maligna and cutaneous metastases of malignant melanoma. IMIQUIMOD and its derivatives such as resIMIQUIMOD (R-848) improve antigen-presentation by DENDRITIC CELLS and also act on B CELLS leading to the synthesis of antibodies such as IgG2a. Furthermore, these agents activate MACROPHAGES and other cells via binding to cell surface receptors, such as TLR7, inducing secretion of proinflammatory CYTOKINES such as IFN- $\alpha$, TNF- $\alpha$ and IL-12. The presence of this CYTOKINE milieu biases towards a Th1-type immune response and has been exploited clinically in the treatment of viral infections (human papillomavirus, herpes simplex virus, molluscum contagiosum). In randomized, double-blind, placebo-controlled stud- 


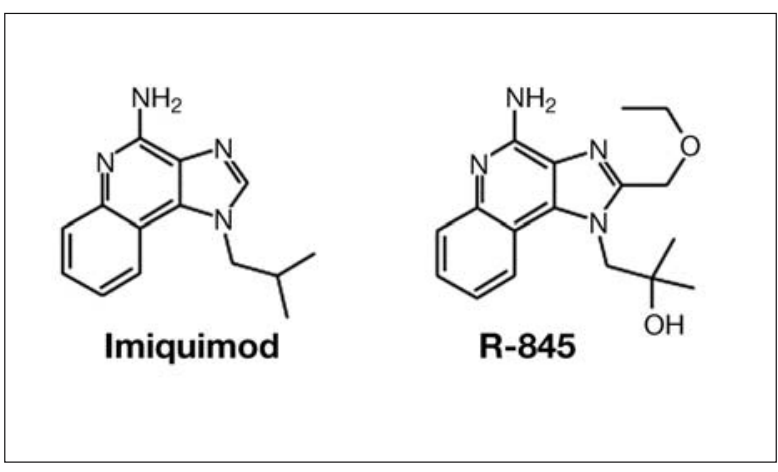

Figure 3. Chemical structure of imiquimod and RESIMIQUIMOD (R-848)

ies, IMIQUIMOD cream has been shown to be significantly effective in eliminating genital warts in patients with clinical, histopathological and polymerase chain reaction confirmed diagnosis of human papilloma virus infection and in treatment of external genital and perianal warts. Topically applied IMIQUIMOD cream reduced wart area in HIV-infected patients (Fig. 3).

\section{Isoprinosine}

ISOPRINOSINE (Inosiplex) is a complex of the $p$ acetamidobenzoate salt of $\mathrm{N}, \mathrm{N}$-dimethylamino-2propanol:inosine in a 3:1 molar ratio. It is a white cystalline powder soluble in water. The inosine portion of ISOPRINOSINE is metabolically labile and half-life in rhesus monkeys has been found to be 3 minutes after intravenous and 50 minutes after oral administration. ISOPRINOSINE has been shown to augment production of CYTOKINES such as IL-1, IL-2 and IFN- $\gamma$. It increases proliferation of LYMPHOCYTES in response to mitogenic or antigenic stimuli, increases active T-cell rosettes and induces T-cell surface markers on prothymocytes. ISOPRINOSINE is currently licensed in Europe for treatment of herpes simplex infections, subacute sclerosing panencephalitis, acute viral encephalitis caused by herpes simplex, Epstein-Barr and measles viruses and for treatment of these viral infections in immunosuppressed patients. ISOPRINOSINE has been reported to have minor CNS depressant but no neuromuscular, sedative, or antipyretic activities in pharmacological studies in animals. In humans ISOPRINOSINE may cause transient nausea and rise of uric acid in serum and urine at high doses.

\section{Emerging therapies with synthetic immunomodulators}

\section{CpG oligodeoxynucleotides}

Over the last few years, nucleotide sequences containing non-methylated cytosine-phosphate-guanosine $(\mathrm{CpG})$ dinucleotides, with flanking regions of two 5' purines and two 3' pyrimidines, have been discovered to play an important role in INNATE IMMUNITY. Effector cells such as MACROPHAGES, DENDRITIC CELLS, B CELLS and NK cells possess pattern recognition receptors. These can bind to the microbe-restricted structure of $\mathrm{CpG}$ motifs and trigger protective immune responses. Although DNA containing $\mathrm{CpG}$ motifs (CpG DNA) evolved as a defense system in eukaryotes to probably protect against infection, it is possible to use CpG DNA as an immunomodulator for therapeutic applications. Most interesting for the induction of antimicrobial effects, CpG DNA triggers a predominantly T helper cell type-1 (Th1) pattern of immune activation. CPG DNA triggers humoral B CELL responses and also activates MONOCYTES and MACROPHAGES to secrete CYTOKINES, especially IL-12, TNF, and IFN. The exact mechanisms by which $\mathrm{CpG}$ DNA induces DENDRITIC CELLS, MACROPHAGES and NK cells to produce immunomodulating CYTOKINES are being elucidated. Recent reports describe molecular mechanisms involved in CpG DNA-mediated activation of immune cells, including cellular recognition of CpG motifs by a member of the TLR family, TLR- 9 . The finding that synthetic oligodeoxynucleotides can mimic the action of bacterial DNA has galvanized research in this area.Administration of synthetic oligodeoxynucleotides (ODNs) containing $\mathrm{CpG}$ motifs [35] has been shown to confer protection or act as an adjuvant in experimental infections by influenza virus, hepatitis virus, Listeria monocytogenes, Francisella tularensis, Trypanosoma cruzi, 
Leishmania and markedly increase resistance against acute poly-microbial sepsis. CpG ODNs have been administered to over 300 humans and found to be well tolerated.

\section{Defensins}

DEFENSINS are a family of structurally related cysteinerich cationic polypeptides of the innate immune system produced in response to microbial infection in humans, animals, insects and plants. Six human $\alpha-$ DEFENSINS have been described; HD1, 2, 3, and 4 are secreted primarily by granulocytes and certain LYMPHOCYTES, whereas HD5 and 6 are expressed by intestinal Paneth cells. $\beta$-DefEnsins are produced in response to microbial infection of mucosal tissue and skin. The unusual circular minidefensins were identified recently in rhesus monkeys. DeFENSINS of vertebrate animals have been reviewed elsewhere [36, 37].

Recent reports have highlighted the anti-HIV-1 activities of DEFENSINS, whose structure and charge resemble portions of the HIV-1 transmembrane envelope glycoprotein gp41. CD8 T LYMPHOCYTES from certain immunologically stable HIV-1-infected individuals secrete soluble factors that suppress HIV-1 replication. CD8 T CELLS from long-term nonprogressors with HIV-1 infection were found to secrete a cluster of proteins identified as $\alpha$-DEFENSIN 1,2 , and 3 on the basis of specific ANTIBODY recognition and amino acid sequencing [38]. It is of interest to note that $\alpha$ DEFENSINS have been shown to effectively suppress HIV replication in vitro about 10 years ago. In earlier studies, synthetic peptides derived from a region of HIV-1 gp41 exhibited a sequence similar to DEFENSINS. Like DEFENSINS, which can insert themselves into lipid bilayers, gp41 peptides have the ability to associate with liposomes and lymphocyte membranes. An antimicrobial peptide homologous to rhesus monkey circular minidefensins ( $\delta$-DEFENSINS) has been prepared by solid-phase peptide synthesis and named "retrocyclin" [39]. Retrocyclin has a remarkable ability to inhibit proviral DNA formation and to protect $\mathrm{CD} 4^{+}$LYMPHOCYTES from in vitro infection by both T-tropic and M-tropic strains of HIV-1. Retrocyclin interferes with an early stage of HIV-1 infection and retrocyclin-like agents might be useful topical agents to prevent sexually acquired HIV-1 infections.

DNA encoding gp120 fused with proinflammatory chemoattractants of immature DENDRITIC CELLS, such as $\beta$-DEFENSIN 2, elicited anti-gp120 antibodies with high titers of virus-neutralizing activity. The use of chemokine fusion constructs with gp140, gp120 linked to the extracellular domain of gp41 via a 14amino acid spacer peptide sequence further augmented immunogenicity. This construct elicited antibodies with more effective neutralizing activity than corresponding constructs expressing gp120 [40]. A fusion polypeptide comprising a DEFENSIN and a viral ANTIGEN and its use for producing an immune response in a subject and for treating viral infections, including HIV infection, have been detailed by the US Department of Health \& Human Services, National Instistute of Health, Office of Technology Transfer. The viral ANTIGEN may be an HIV ANTIGEN, and the DEFENSIN may be an $\alpha$-DEFENSIN or a $\beta$-DEFENSIN. It is interesting to note that the peptides derived from HIV-1 envelope glycoproteins corresponding predominantly to membrane-active domains of gp120 and gp41can exert antimicrobial activity against laboratory strains of Escherichia coli and Listeria monocytogenes. Human $\beta$-DEFENSIN-3 (h $\beta D-3$ ) and fragments of $h \beta D-3$ for the treatment of various infections, particularly respiratory infections, have been described. The peptides are claimed to be of use in the treatment of infection caused by Pseudomonas aeruginosa and Burkholderia cepacia, other gramnegative and gram-positive bacterial infections and yeast infections. The peptides were synthesized and tested with a range of bacteria such as Enterococcus faecalis, Escherichia coli, Klebsiella pneumoniae, Staphylococcus aureus, Streptococcus pneumoniae, P. aeruginosa and B. cepacia.

\section{Methyl inosine monophosphate (MIMP)}

MIMP is a newer generation thymomimetic purine immunomodulator capable of enhancing a wide variety of immune responses. It can induce in human prothymocytes the expression of T lymphocyte differentiation markers (CD3,CD4,CD8) and IL-2 receptors 
(CD25), enhance mitogen-induced proliferation of LYMPHOCYTES, augment IgM plaque-forming cells, induce delayed-type hypersensitivity and normalize an impaired response to IL-2 and suggest MIMP as a potential IMMUNOTHERAPY for early HIV infection and for inducing protective effects against human viruses. Intranasal administration of MIMP improves survival rates and incorporation of MIMP in squalanesaline emulsion confers complete protection against influenza infection. Complete survival and reduction of viral load after influenza challenge infection suggests effective stimulation by MIMP of protective responses against influenza virus [41].

\section{Microbial-derived synthetic immunomo- dulators}

Cell walls of gram-negative bacteria contain endotoxic lipopolysaccharide which is a potent stimulator of the immune system, even in nanogram quantities. Clinical application of endotoxin has, however, been hampered as it plays a major role in the pathophysiology of gram-negative bacterial sepsis. Concerted efforts have been made to modify endotoxin for possible therapeutic use in humans. Investigations on endotoxic lipopolysaccharide have led to the identification of lipid A as an important constituent of the endotoxin molecule capable of exhibiting various immunopharmacological activities of bacterial lipopolysaccharide. During the last ten years, selective reduction of the toxicity of endotoxin whilst retaining its beneficial adjuvant property has been achieved by effective chemical treatment and by synthesis of nontoxic lipid A analogues.

\section{Monophosphoryl lipid A}

Prophylactic administration of monophosphoryl lipid A (MPL), a nontoxic derivative of endotoxin, has been shown to mitigate the sepsis syndrome by reducing chemokine production of pulmonary and hepatic MIP-1 $\alpha$,MIP-1 $\beta$, MIP-2, and MCP-1 mRNA and attenuating the production of proinflammatory CYTOKINES. MPL has been used as an adjuvant with leishmanial, malarial, tuberculosis and cancer ANTIGENS. It has been administered to over thirty thou- sand humans and is a component of a melanoma Melacine vaccine which is approved for use in Canada.

\section{$\mathrm{OM}-174$}

IMMUNOMODULATOR OM-174 is a chemically defined new generation lipid A analogue. Animal experiments using aerosol influenza virus showed that the survival in the group receiving H1N1 SUBUNIT VACCINE alone could be improved after the booster immunization. In contrast, the vaccine adjuvanted with OM-174 could confer complete protection already after the primary immunization. OM-174 has shown adjuvant activity with several microbial ANTIGENS and has already been administered intramuscularly to humans in a limited number of trials.

\section{Murabutide}

Muramyl peptides (MDP) represent structures that are essential for mycobacterial adjuvanticity. MURABUTIDE, an MDP analogue, significantly reduced reduced CD4 and CCR5 receptors, secreted high levels of $\beta$-CHEMOKINES and suppressed HIV-1viral replication in acutely infected MONOCYTE-derived MACROPHAGES and DENDRITIC CELLS. Clinical TOLERANCE and biological effects of IMMUNOTHERAPY with the synthetic immunomodulator MURABUTIDE have been evaluated in HIV-1 patients [42]. MURABUTIDE recipients showed a significant increase in CD4 cells and platelet counts in HIV-1 patients with weak immune reconstitution and ineffective virus suppression following long-term HAART therapy.

\section{Microbial immunomodulators}

\section{Probiotics}

Ingestion of living microorganisms for attaining beneficial effects on health has been termed as a probiotic approach which is gaining increasing popularity. Several fortified products are available to consumers. Probiotics have been used for conditions such as diarrhea, candidal vaginitis and urinary tract infections [43]. Whole microbes such as Lactobacil- 
lus casei, Bifidobacterium species and Saccharomyces boulardii have been used successfully to prevent antibiotic-associated diarrhea, and to treat other diarrheal illnesses caused by bacteria [44]. Bifidobacterium breve has been shown to augment ANTIBODY production and induce significant reduction in the frequency of rotavirus shedding in stool samples of infants [45]. The major Lactobacillus species present in the human gastrointestinal mucosa, L. plantarum, L. rhamnosus and L. paracasei, have been found to be potent stimulators of INTERLEUKIN-12 [46].

\section{Bacterial extracts}

BACTERIAL EXTRACTS have been widely used as IMMUNOMODULATORS to prevent recurrent infections of the respiratory tract. Several preparations containing bacterial lysates (e.g., Broncho-Munal ${ }^{\circledR}$, Luivac $^{\circledR}$ ) are licensed for use in Europe. There exists a large body of primary literature on placebo-controlled and double-blind clinical trials which have been conducted with these agents. The current concept of the mucosal immune system postulates that stimulation of the gut-associated lymphoid tissue can lead to the induction of a generalized response by the whole mucosal-associated lymphoid tissue. Patients, most often children, having recurrent episodes of infections of the respiratory tract and of the ear, nose and throat have been treated with oral bacterial lysates. In most cases the frequency and the severity of infections was reported to be reduced and both the physician and the patient considered the treatment to be beneficial. It is of interest that many studies have reported a decrease in antibiotic consumption in bacterial lysate-treated patients. A multicenter study with oral bacterial lysate immunomodulator LW 50020 involving 4965 recurrent respiratory tract infection patients in 14 countries in Europe, Latin America, and Asia has recently been conducted. An overall reduction of at least $50 \%$ in the number,severity, and duration of respiratory tract infections, the number of antibiotic and symptomatic treatments, and the number of days absent from school or work was reported [47]. Hemodialysis patients suffer from several immune defects which increase their risk of developing bacterial infections, particularly of the respiratory tract. In a double-blind placebo-controlled prospective study, oral IMMUNOTHERAPY with an immunomodulating bacterial extract significantly reduced the number of patients with respiratory tract infections and consequently the number of antibiotic treatment courses [48].

\section{Glucans}

Immunomodulating polysaccharides, poly-(1-6)- $\beta$ glucotriosyl-(1-3)- $\beta$-glucopyranose, PGG-GLUCAN, marketed as Betafectin, have been found to be useful in prevention of mortality and abscess formation associated with experimental intraabdominal sepsis in the absence of antimicrobial therapy. The safety and EFFICACY of PGG-GLUCAN in surgical patients at high risk for postoperative infection who underwent major thoracic or abdominal surgery showed that PGG-GLUCAN had significantly fewer infectious complications, decreased intravenous antibiotic requirement and shorter intensive care unit length of stay. Prophylaxis to prevent staphylococcal wound infection could be synergistically enhanced by adding PGG-GLUCAN to the antibiotic cefazolin. Perioperative administration of PGG-GLUCAN reduced serious postoperative infections or death by $39 \%$ after high-risk noncolorectal operations.

LENTINAN is chemically well defined as 1-3- $\beta$-D-glucan with 1-6- $\beta$-D-glucopyranoside branches and is isolated from an edible Japanese mushroom Lentinus edodes (Shiitake mushroom) and has immunomodulating properties. It is licensed as an adjunctive for antitumor therapy in Japan. Experimental studies have demonstrated that LENTINAN can confer protection against influenza virus, Listeria and prevent relapse of Mycobacterium tuberculosis. Yeast glucan could enhance resistance against herpes simplex virus types I and II, and murine hepatitis virus, and polysaccharide schizophyllan could confer protection on mice against lethal Sendai virus infection. $S$. cervisiae glucan induced nonspecific resistance against K. pneumoni$a e$ infection and yeast glucan can protect patients from sepsis, bacteremia and peritonitis resulting from Escherichia coli, Staphylococcus aureus and Pseudomonas aeruginosa infections. Yeast glucan 
can prolong survival against parasitic infections by Plasmodium berghei and Leishmania donovani and exert antifungal activity against Candida, Cryptococcus and Sporotichum. Phase I/II placebocontrolled trials with LENTINAN Or LENTINAN in combination with didanosine in HIV-positive patients showed a trend toward increases in CD4 cells and in NEUTROPHIL activity.

\section{Perspective}

Preventing existing and emerging infectious diseases is a multidisciplinary and multifaceted endeavor.The traditional treatment of infections has focused on antimicrobial agents and on the induction of specific immune defenses. The forefront of immunotherapeutics in the coming decades will be on the modulation of the host response against infectious diseases. Selective stimulation of discrete lymphocyte subpopulations important in protective effector mechanisms against a given infection by suitable IMMUNOMODULATORS will play an increasingly important role. Polarized type- 1 and type- 2 immune responses can be readily achieved, irrespective of the genetic bias of the host and of the nature of the protein ANTIGEN, through the choice of appropriate IMMUNOMODULATORS. The major promise of new IMMUNOMODULATORS lies in their broad activity against diverse microbial pathogens and in a mode of action that is distinctly different from the direct microbicidal action of established antibiotics, antivirals and antifungals. Adjuvant CYTOKINES such as IL-2, IFN- $\gamma$, and GM-CSF, can be useful for shortening the duration of treatment. It is now also possible to control the deleterious overproduction of inflammatory CYTOKINES observed in certain disease states with several IMMUNOMODULATORY agents. Activation of innate non-antigen-specific host defenses by recently recognized IMMUNOMODULATORY agents such as CPG DNA will increasingly find a beneficial role in the prevention of infections. It is noteworthy that around 60 biotechnology protein drugs, including recombinant proteins and MONOCLONAL ANTIBODIES, that are currently available represent over a quarter of the drugs introduced worldwide recently. New methodologies currently available have the potential to identify novel targets and foster the development of individually tailored IMMUNOMODULATORY drug treatments. The human genome project has indicated that the human genome contains 30,000-40,000 proteinencoding genes with an estimated 90,000 or more distinct proteins. It would require only a fraction, 1 or $2 \%$, of these proteins to be developed as drugs to be able provide hundreds of novel therapeutic agents. IMMUNOMODULATORS are becoming a viable adjunct to established modalities offering a novel approach for the treatment of infectious diseases in the coming decades of the 21 st century.

\section{Summary}

The availability of a vast array of recombinant and synthetic IMMUNOMODULATORS is a significant milestone toward the development of effective therapies for infectious diseases. This is evinced by licensing of several recombinant human CYTOKINES, including COLONY-STIMULATING FACTORS, INTERLEUKINS, INTERFERONS and erythropoietin, for clinical use in patients. Diverse combinations with INTERFERONS and other CYTOKINES for the treatment of various infections have been proposed. Others, including various CHEMOKINES, synthetic CpG oligodeoxynucleotides and glucans, are extensively being investigated in clinical and preclinical studies. Considerable advances have been made on compounds exhibiting CYTOKINE inhibitory properties useful for new treatments of infectious and inflammatory diseases. Many of the major developments and current trends are highlighted in this review. Novel strategies based on the engineering of CYTOKINES and inhibitors are poised to revolutionize therapeutic options contingent upon scientific evidence rather than dictates of discursive empiricism in the coming decades.

\section{Recommended websites}

The Cleveland Clinic (2002) Biologictherapy.org: http://www.biologictherapy.org (Accessed March 2005) Horst Ibelgaufts' COPE (2003) Cytokines Online Pathfinder Encyclopaedia: http://www.copewithcytokines.de (Accessed March 2005) 
Beta Glucan Research Organization (2003) Non-Commercial Beta Glucan Research Site Sorted by Health Condition: http://www.betaglucan.org (Accessed March 2005)

\section{References}

1 Ksiazek TG, Erdman D, Goldsmith C, Zaki SR, Peret T, Emery S, Tong S, Urbani C, Comer JA, Lim W et al (2003) A novel coronavirus associated with severe acute respiratory syndrome. $N$ Engl $J$ Med 348: 1953-1966

2 Taylor PW, Stapleton PD, Paul LJ (2002) New ways to treat bacterial infections. Drug Discov Today 7: 1086-1091

3 Amyes SGB (2000) The rise in bacterial resistance is partly because there have been no new classes of antibiotics since the 1960s. Br Med J 320: 199-200

4 Hengel H, Masihi KN (2003) Combinatorial immunotherapies for infectious diseases. Int Immunopharmacol 3: 1159-1167

5 Masihi KN (2003) Concepts of immunostimulation to increase antiparasitic drug action. Parasitol Res 90 (Suppl 2): S97-S104

6 Masihi KN (2001) Fighting infection using immunomodulatory agents. Expert Opin Biol Ther 1: 641-653

7 Koliouskas D, Sidiropoulos I, Masmanidou M, Dokas S, Ziakas A (2002) Comparative analysis of Peg-interferon alpha-2b and lamivudine in the treatment of chronic hepatitis B patients: preliminary results.J Hepatol 36 : 237-238

8 Santantonio T, Anna N, Sinisi E, Leandro G, Insalata M, Guastadisegni A, Facciorusso D, Gravinese E, Andriulli A, Pastore G (2002) Lamivudine/interferon combination therapy in anti-HBe positive chronic hepatitis B patients: a controlled pilot study.J Hepatol 36: 799-804

9 Billich A (2002) Thymosin alpha1. SciClone Pharmaceuticals. Curr Opin Investig Drugs 3: 698-707

10 Sugahara S, Ichida T,Yamagiwa S, Ishikawa T, Uehara K, Yoshida Y,Yang XH, Nomoto M, Watanabe H,Abo T et al (2002) Thymosin-[alpha] 1 increases intrahepatic NKT cells and CTLs in patients with chronic hepatitis B. Hepatol Res 24: 346-354

11 Pimstone NR, Canio JB, Chiang MH (2001) Ribavirin/ interferon-2b therapy is very effective in the treatment of chronic hepatitis $\mathrm{C}$ genotype 2 and 3 patients who have failed to respond virologically to IFN monotherapy. Gastroenterology 120: A-382

12 Luxon BA, Grace M,Brassard D,Bordens R (2002) Pegylated interferons for the treatment of chronic hepatitis C infection. Clin Ther 24: 1363-1383

13 Rajender R, Modi MW,Pedder S (2002) Use of peginterferon alfa-2a (40 KD) (Pegasys(R)) for the treatment of hepatitis C.Adv Drug Deliv Rev 54:571-586

14 Welch W,Foote M (1999) The use of Filgrastim in AIDSrelated neutropenia. J Hematother Stem Cell Res 8 (Suppl 1):S9-S16

15 Kuritzkes DR (1999) Clinical experience with Filgrastim in AIDS.J Hematother Stem Cell Res 8 (Suppl 1): S17-S19

16 Dubreuil-Lemaire ML, Gori A, Vittecoq D, Panelatti G, Tharaux F, Palisses R, Gharakhanian S, Rozenbaum W; GCS 309 European Study Group (2000): Lenograstim for the treatment of neutropenia in patients receiving ganciclovir for cytomegalovirus infection: a randomised, placebo-controlled trial in AIDS patients. Eur J Haematol 65: 337-343

17 Rodriguez-Adrian LJ, Grazziutti ML, Rex JH,Anaissie EJ (1998) The potential role of cytokine therapy for fungal infections in patients with cancer: is recovery from neutropenia all that is needed? Clin Infect Dis 26: 1270-1278

18 Masihi KN (2003) Progress on novel immunomodulatory agents for HIV-1 infection and other infectious diseases. Expert Opin Ther Patents 13: 867-882

19 Pett SL, Emery S (2001) Immunomodulators as adjunctive therapy for HIV-1 infection.J Clin Virol 22: 289-295

20 David D, Nait-Ighil L, Dupont B, Maral J, Gachot B, Theze J (2001) Rapid effect of interleukin-2 therapy in human immunodeficiency virus-infected patients whose CD4 cell counts increase only slightly in response to combined antiretroviral treatment.J Infect Dis 183: 730-735

21 Aquaro S, Calio R, Balzarini J, Bellocchi MC, Garaci E, Perno CF (2002) Macrophages and HIV infection: therapeutical approaches toward this strategic virus reservoir. Antiviral Res 55: 209-225

22 Deng H,Liu R, Ellmeier W, Choe S, Unutmaz D, Burkhart M, Di Marzio P, Marmon S, Sutton RE, Hill CM et al (1996) Identification of a major co-receptor for primary isolates of HIV-1. Nature 381: 661-666

23 Dragic T (2001) An overview of the determinants of 
CCR5 and CXCR4 co-receptor function.J Gen Virol 82: 1807-1814

24 Lehner T (2002) The role of CCR5 chemokine ligands and antibodies to CCR5 coreceptors in preventing HIV infection. Trends Immunol 23: 347-351

25 Nansen A, Christensen JP, Andreasen SO, Bartholdy C, Christensen JE, Thomsen AR (2002) The role of CC chemokine receptor 5 in antiviral immunity. Blood 99: 1237-1245

26 Alkhatib G, Combadiere C, Broder CC, Feng Y, Kennedy PE, Murphy PM,Berger EA (1996) CC CKR5: a RANTES, MIP-1alpha, MIP-1beta receptor as a fusion cofactor for macrophage-tropic HIV-1.Science 272: 1955-1958

27 Blanpain C, Libert F, Vassart G, Parmentier M (2002) CCR5 and HIV infection. Receptors Channels 8: 19-31

28 Lusso P (2002) HIV and chemokines: implications for therapy and vaccine. Vaccine 20: 1964-1967

29 Scozzafava A, Mastrolorenzo A, Supuran CT (2002) Non-peptidic chemokine receptors antagonists as emerging anti-HIV agents. J Enzyme Inhib Med Chem 17: 69-76

30 Verani A, Lusso P (2002) Chemokines as natural HIV antagonists. Curr Mol Med 2: 691-702

31 Onuffer JJ, Horuk R (2002) Chemokines, chemokine receptors and small-molecule antagonists: recent developments. Trends Pharmacol Sci 23: 459-467

32 Regoes RR, Bonhoeffer SEBA (2002) HIV coreceptor usage and drug treatment.J Theor Biol 217: 443-457

33 Polo S, Nardese V, De Santis C, Arcelloni C, Paroni R, Sironi F,Verani A, Rizzi M, Bolognesi M, Lusso P (2000) Enhancement of the HIV-1 inhibitory activity of RANTES by modification of the N-terminal region: dissociation from CCR5 activation. Eur J Immunol 30: 3190-3198

34 Stanley MA (2002): Imiquimod and the imidazoquinolones: mechanism of action and therapeutic potential. Clin Exp Dermatol 27: 571-577

35 Krieg AM, Davis HL (2001) Enhancing vaccines with immune stimulatory CpG DNA. Curr Opin Mol Ther 3: 15-24

36 Lehrer RI, Ganz T (2002) Defensins of vertebrate animals. Curr Opin Immunol 14: 96-102

37 Raj PA, Dentino AR (2002) Current status of defensins and their role in innate and adaptive immunity. FEMS Microbiol Lett 206: 9-18

38 Zhang L,Yu W, He T,Yu J, Caffrey RE, Dalmasso EA, Fu S, Pham T, Mei J, Ho JJ et al (2002) Contribution of human alpha-defensin 1,2, and 3 to the anti-HIV-1 activity of CD8 antiviral factor. Science 298: 995-1000

39 Cole AM, Hong T, Boo LM, Nguyen T, Zhao C, Bristol G, Zack JA, Waring AJ,Yang OO, Lehrer RI (2002) Retrocyclin: a primate peptide that protects cells from infection by T- and M-tropic strains of HIV-1.Proc Natl Acad Sci USA 99: 1813-1818

40 Biragyn A, Belyakov IM, Chow YH, Dimitrov DS, Berzofsky JA, Kwak LW (2002) DNA vaccines encoding human immunodeficiency virus-1 glycoprotein 120 fusions with proinflammatory chemoattractants induce systemic and mucosal immune responses. Blood 100: 1153-1159

41 Masihi KN, Hadden JW (2002) Protection by methyl inosine monophosphate (MIMP) against aerosol influenza virus infection in mice. Int Immunopharmacol 2: 835-841

42 Bahr GM (2003) Non-specific immunotherapy of HIV1 infection: potential use of the synthetic immunodulator murabutide.J Antimicrob Chemother 51: 5-8

43 Mombelli B, Gismondo M (2000) The use of probiotics in medical practice. Int $J$ Antimicrob Agents 16: 531536

44 Cross ML (2002) Microbes versus microbes: immune signals generated by probiotic lactobacilli and their role in protection against microbial pathogens. FEMS Immunol Med Microbiol 34: 245-253

45 Yasui H, Shida K, Matsuzaki T, Yokokura T (1999) Immunomodulatory function of lactic acid bacteria. Antonie Van Leeuwenhoek 76: 383-389

46 Hessle C, Hanson LÅ, Wold AE (1999) Lactobacilli from human gastrointestinal mucosa are strong stimulators of IL-12 production. Clin Exp Immunol 116: 276-282

47 Grevers G, Palacios OA, Rodriguez B, Abel S, van Aubel A (2000) Treatment of recurrent respiratory tract infections with a polyvalent bacterial lysate: results of an open, prospective, multinational study. Adv Ther 17: 103-116

48 Tielemans C, Gastaldello K, Husson C, Marchant A, Delville JP,Vanherweghem JL, Goldman M (1999) Efficacy of oral immunotherapy on respiratory infections in hemodialysis patients: a double-blind, placebo-controlled study. Clin Nephrol 51: 153-160 